\title{
Pratiques informationnelles et identités numériques
}

Information Practices and Digital Identities

\section{Fanny Georges}

\section{CpenEdition}

\section{Journals}

Édition électronique

URL : http://journals.openedition.org/edc/2226

DOI : $10.4000 /$ edc.2226

ISSN : 2101-0366

Éditeur

Université de Lille

Édition imprimée

Date de publication : 1 décembre 2010

Pagination : 105-120

ISBN : 978-2-917562-04-8

ISSN : $1270-6841$

Référence électronique

Fanny Georges, «Pratiques informationnelles et identités numériques », Études de communication [En ligne], 35 | 2010, mis en ligne le 01 décembre 2012, consulté le 10 décembre 2020. URL : http:// journals.openedition.org/edc/2226 ; DOI : https://doi.org/10.4000/edc.2226

Ce document a été généré automatiquement le 10 décembre 2020.

(c) Tous droits réservés 


\title{
Pratiques informationnelles et identités numériques
}

\author{
Information Practices and Digital Identities
}

Fanny Georges

1 L'usage des outils de réseaux sociaux par les bibliothèques commence à se développer. Les lecteurs utilisent les fonctionnalités classiques de construction d'un réseau d'« amis » en se socialisant par la production de commentaires et d'appréciations quantifiées sur les collections (UCL, 2008 ; Suraweera et al., 2010). L'effort d'intégration de ces outils dans les bibliothèques présente un fort potentiel : valorisation des collections, ouverture à de nouveaux publics, participation des lecteurs à la documentarisation, voire à la collecte de documents numériques lorsque celle-ci est envisagée ${ }^{1}$. Le partage de médias et son actualisation par l'écriture de commentaires pourraient engendrer une dynamique de partage de connaissances mais également une dynamique sociale qui pourrait aller jusqu'à changer l'image parfois « déconnectée » des nouvelles réalités numériques qu'ont parfois les bibliothèques chez ces populations (Suraweera et al., 2010).

2 Si les bénéfices pour les bibliothèques sont identifiés, la réussite d'un tel projet est encore difficile à évaluer : l'usager de la génération " Google » va-t-il nourrir un intérêt pour ces réseaux ad-hoc? (Silipigni et Dickey, 2010). Les réseaux trop spécifiques se heurtent à une difficulté immédiate : les utilisateurs enclins à les utiliser sont généralement inscrits sur des réseaux sociaux ouverts rassemblant des communautés plus importantes, comme Facebook, Myspace, Viadeo, Linkedin ou encore Academia.edu. Dès lors, s'inscrire dans un réseau social ad hoc peut apparaître trop coûteux pour le lecteur/utilisateur en regard du bénéfice immédiat envisagé : l'usage de réseaux sociaux externes paraît plus pertinent (Suraweera et al., 2010).

3 Quelles peuvent être les motivations des utilisateurs à utiliser un outil de réseau social dans le cadre de leurs activités de lecteur? Le partage des connaissances et la participation bénévole à un projet culturel sont des motivations qui pourraient être citées en premier lieu ; mais au-delà, la socialisation par cet investissement personnel de lecteur engendre, dans les réseaux sociaux, une problématique bien spécifique, celle de la 
présentation de soi. En effet, les réseaux sociaux en ligne ont en commun de relier les utilisateurs par leur profil personnel ; ils sont de plus considérés par les adolescents comme participant de leur socialisation globale (Walther et al., 2008) : commenter des documents en ligne, qu'il s'agisse de photos de soirées dans Facebook, d'albums de musique dans Myspace ou d'articles dans Academia.edu, participe de la construction de l'image de soi informatisée (le profil).

4 Au centre de ce phénomène apparaît la figure de l'être humain comme document: fournissant des informations sur les documents qu'il consulte, partageant à la collecte par l'enregistrement de séquences de jeux vidéo ou le partage de vidéos (Suraweera et al., 2010), l'utilisateur construit son profil. Ce dernier devient en conséquence un objet documentaire agrégeant des traces d'usage dont les éléments partagés et indexés (livre favori, groupes d'utilisateurs) apparient les individus. Bien que ces derniers ne réfèrent pas à l'utilisateur, ils font partie de la présentation de soi (au sens de Goffman) informatisée : les signes autoréférentiels s'intriquent aux documents produits, consultés et diffusés par l'utilisateur. L'ensemble peut faire l'objet de pratiques informationnelles ayant pour finalité de rencontrer des individus partageant les mêmes centres d'intérêt ou mêmes objets. Ces pratiques peuvent poser question dans le cadre des usages des lecteurs : dispersion? Superficialité ? La socialisation informatisée a-t-elle une place dans les bibliothèques? Quels sont l'intrication des médias partagés à l'identité documentée ?

5 La conception d'un projet d'intégration des réseaux sociaux en ligne dans les bibliothèques doit tenir compte du caractère documenté des identités informatisées. Cet article présente un commencement de réflexion à ce sujet, en proposant une relecture synthétique de nos travaux sur l'identité numérique (Georges, 2009 ; Georges et al., 2010) à la lumière des pratiques informationnelles ayant pour objet les composantes de l'identité numériques de l'individu-document (Ertzscheid, 2009). En conclusion sont proposées des pistes d'étude des usages et de conception de ces outils pour les bibliothèques.

\section{Pratique informationnelle des individus-documents}

6 Les travaux sur les recherches documentaires en sciences de l'information ont connu plusieurs paradigmes (Henneron et al., 1997) : le paradigme classique orienté système, le paradigme cognitif orienté utilisateur, qui place l'utilisateur et son interaction avec le système au centre du système d'information, le paradigme social orienté usages, qui "considère que l'attention doit être portée sur les besoins réels de l'usager et son environnement » et propose « une modélisation des usagers et de leurs comportements » (Chaudiron et Ihadjadene, 2002); enfin, le paradigme activité (Choo, 1996; Thivant et Bouzidi, 2005) qui «considère que l'activité influence plus ou moins directement les pratiques de recherche et d'utilisation de l'information» (Thivant et Bouzidi, $2005: 15$ ). Ces différents paradigmes permettent chacun de mettre en lumière des dimensions spécifiques d'un objet de recherche; dans leur succession chronologique, le concept de pratique informationnelle se complexifie de la prise en compte des cadres de l'expérience de recherche d'information. Ainsi, selon le paradigme orienté usages, la pratique informationnelle "peut être considérée comme l'ensemble des actions et des choix de l'individu lors d'une phase de recherche d'information provoquée par un besoin d'information » (Sarméjeanne, 2001). Le paradigme activité approfondit la prise en compte des besoins, qu'il reformule et développe en termes de nécessités, anomalies, manques et resitue dans la finalité d'une action dont le résultat de la recherche vise à permettre la 
mise en œuvre. En s'appuyant sur ces travaux, on peut définir la notion de pratique informationnelle dans le domaine de la communication intersubjective médiée par ordinateur comme l'ensemble des actions et des choix d'un utilisateur opérés lors d'une phase de recherche d'information sur un autre utilisateur ou sur lui-même. Les finalités de la recherche d'informations sur ces deux objets sont dans le premier cas le jugement d'une personne (Walther et al., 2008) et dans le second cas le jugement de l'image qu'un autre utilisateur peut se faire de soi-même. Les dispositifs du web social nécessitant en principe de créer soi-même un compte pour accéder à des informations sur d'autres personnes, il en résulte que l'ensemble des utilisateurs est documenté. Les individus sont des documents : l'être humain est devenu indexable et peut faire l'objet de pratiques informationnelles, comme « objet-documentaire » (Ertzscheid, 2009).

7 Au cœur de la pratique informationnelle des individus-documents, figure la problématique de l'identité numérique. Cette expression, sujette à controverses et divergences terminologiques, est définie par 0 . Ertzscheid dans une perspective documentaire, comme :

la collection des traces (écrits, contenus audio ou vidéo, messages sur des forums, identifiants de connexion, actes d'achat ou de consultation...) que nous laissons derrière nous, consciemment ou inconsciemment, au fil de nos navigations sur le réseau et de nos échanges marchands ou relationnels dans le cadre de sites dédiés. Cet ensemble de traces, une fois qu'il apparaît "remixé" par les moteurs de recherche ou les sites de réseaux sociaux, définit alors un périmètre qui est celui de notre réputation numérique (Ertzscheid, $2009: 35$ ).

8 Cette définition présente le cadre humain et technologique de la situation de présentation de soi en ligne : produite par l'activité humaine, l'identité numérique est la somme des traces emprises dans le support hypermédiatique, dans la mesure où elles donnent prise à une production de signification de la part d'un tiers consultant la page de profil. La collection et l'indexation de ces traces est moins une finalité de l'individu producteur que du système informatique (moteurs de recherche, sites de réseaux sociaux). Le système, décompose, recompose, filtre et enregistre les informations, quelle qu'en soit la volonté de l'utilisateur: les pratiques informationnelles ayant pour objet l'individu-document portent donc sur une collection de traces recomposées par le système local (par exemple: Google, Facebook) : cette recomposition peut donner lieu à une perte de maîtrise de l'utilisateur sur ses données personnelles.

O. Ertzscheid distingue trois processus dans l'économie informationnelle de l'identité numérique (fig.1), montrant que l'homme dispose d'une identité dont il n'est plus le propriétaire car il en contrôle moins la visibilité : l'Ego-documentation (processus de documentation identitaire autoréférentielle maîtrisée sur soi), l'Inter-documentation (processus d'appariement des individus par les fonctionnalités de mise en relation) et l' Alter-documentation (processus par lequel les documents autoréférentiels s'émancipent du champ de maîtrise de l'individu pour apparaître dans des espaces qui ne lui sont pas accessibles). Cette troisième composante met en exergue le rôle des documents produits et partagés par les individus comme participant d'une échappée de leurs productions vers des espaces non maîtrisés par eux. Les commentaires postés ayant pour objet les collections des bibliothèques, s'inscrivent à la fois dans la collection de traces autoréférentielles dans la mesure où elles pointent implicitement sur une subjectivité traçable (celle du lecteur), tout en s'en émancipant vers l'espace des connaissancesdocuments partagées. 
Figure $1:$ «Carré sémiotique de nos identités documentaires » (Ertzscheid, 2009)

Image 2000000900003E1B00002502428353F9.wmf

Ce modèle sémiotique des identités documentaires montre comment les individusdocuments sont recomposés par le système dans les profils utilisateur de sorte, d'une part, à faire émerger des signes communs entre les personnes et favoriser la mise en relation interpersonnelle, et, d'autre part, à faciliter les pratiques informationnelles par des fonctionnalités liées à l'indexation des individus-documents dans les moteurs de recherche, dont font partie les documents recherchés, partagés et commentés. Comment les outils de réseaux sociaux en ligne informent-ils la présentation des individus et quel rôle confèrent-ils aux documents produits?

\section{Mesure du processus d'alter-documentation}

11 Nous avons isolé les informations qui composent les individus-documents et les avons recomposées dans un modèle original de l'Identité numérique mettant en évidence le processus d'alter-documentation en trois degrés d'emprise du système sur la présentation de l'individu (fig. 2 et 3).

L'identité numérique est définie au sens donné par O. Ertzscheid comme collection de traces agrégées par le système. Le modèle se compose de trois catégories d'informations : l'identité déclarative, l'identité agissante et l'identité calculée (fig. 2 et 3). L'identité déclarative se compose de données saisies directement par l'utilisateur, notamment au cours de la procédure d'inscription au service (exemple : nom, centres d'intérêt, amis). L' identité agissante est constituée des notifications du système concernant les activités de l'utilisateur (exemple dans l'historique de Facebook : "X et Y sont désormais amis », "X a utilisé l'application $\mathrm{Z} »)$. L'identité calculée se compose de chiffres, produits du calcul du système, qui sont dispersés sur le profil de l'utilisateur (exemple: nombre d'amis, de groupes). Ces trois dimensions mettent en évidence trois traitements différents d'une même information, selon un degré croissant d'emprise du système sur la présentation de soi (Georges, 2009) et donc un processus d'alter-documentation croissant.

Figure 2 : Représentation de soi et identité numérique

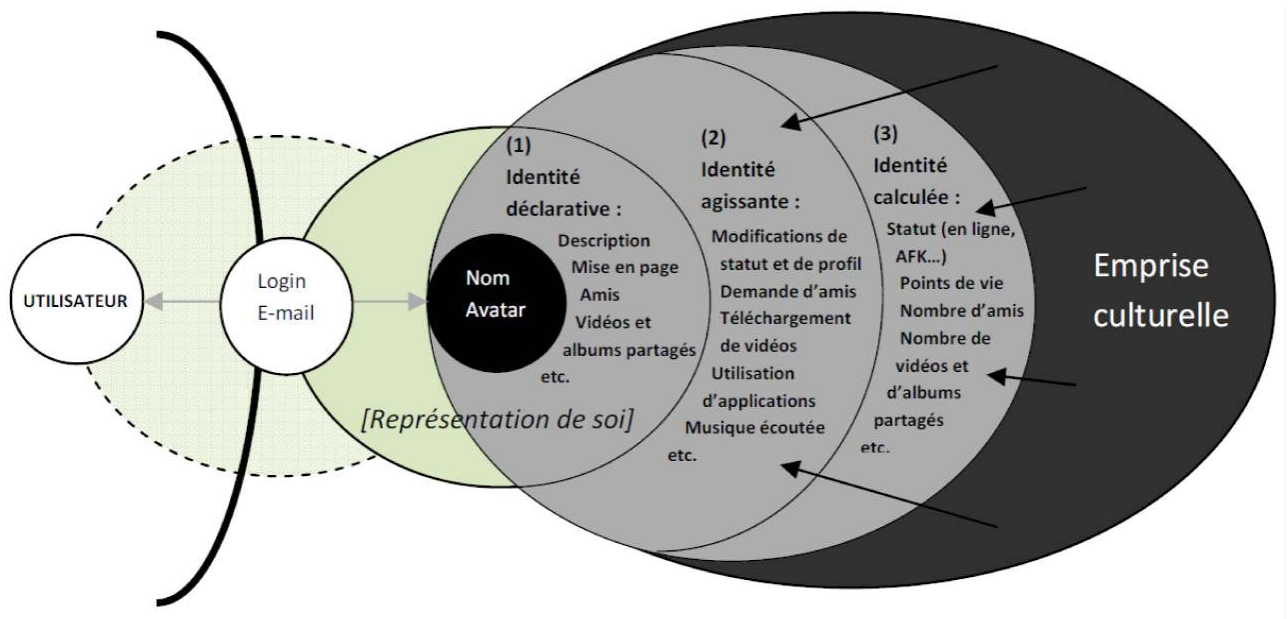




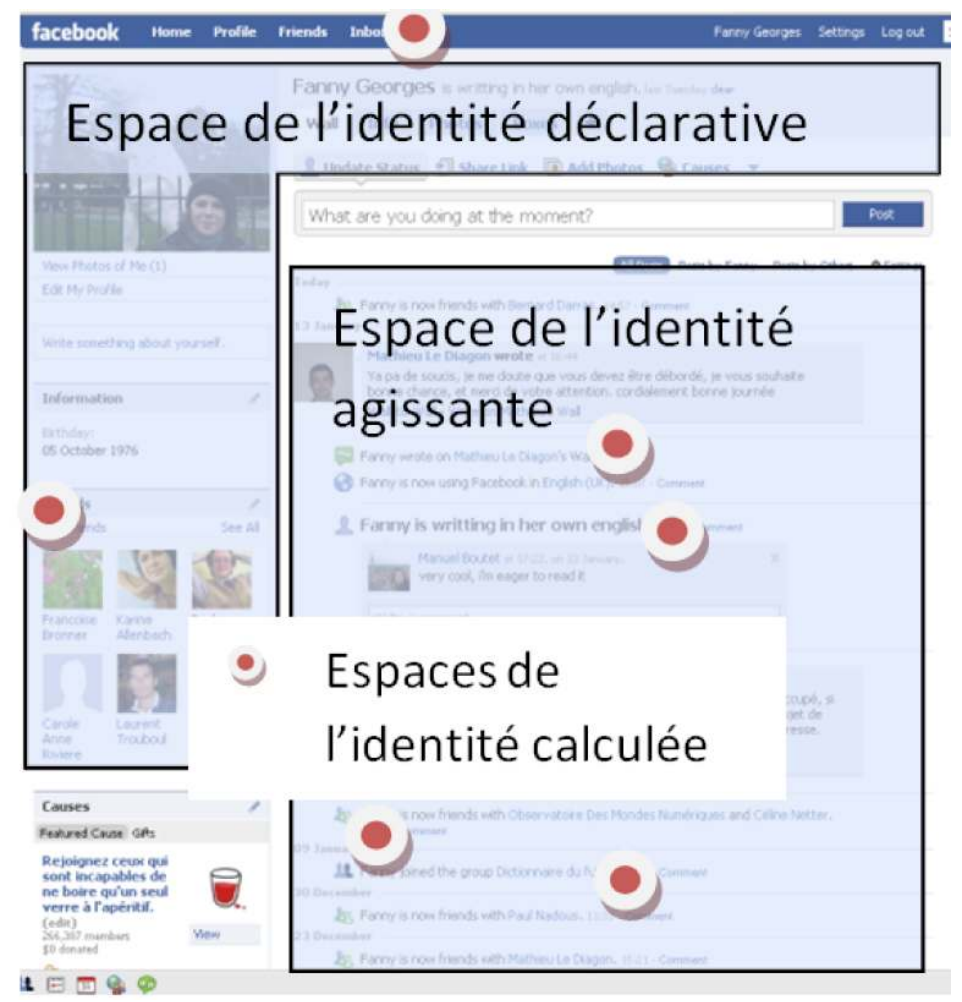

Plusieurs méthodes d'application du modèle présenté ont été proposées en vue de l'analyse quantifiée de l'alterdocumentation.

Nous avons premièrement questionné le changement de paradigme identitaire, du premier web dominé par l'identité déclarative, au web social dominé par les identités agissante et calculée : les utilisateurs qui ne remplissent aucun champ déclaratif (identité déclarative) ont-ils une identité distinctive ? Les résultats ont montré que les utilisateurs qui ne remplissent aucun champ (cf. population cachée : fig. 4, ligne 2) ont une identité distinctive par l'identité agissante et calculée, tout autant que les utilisateurs qui remplissent la majorité des champs disponibles (cf.population hypervisible: fig. 4, ligne 3) : Facebook contourne les stratégies de préservation des données personnelles en développant une présentation de soi automatisée, fondée sur le relevé, par le système, des activités en ligne, et par une quantification de certaines informations qui rendent les profils comparables du point de vue de la sociabilité (comparaison du nombre d'amis, du nombre de commentaires reçus). L'identité numérique de l'utilisateur du web 2.0 repose davantage sur les traces de ses activités en ligne (identités agissante et calculée) que sur les informations qu'il déclare volontairement pour se présenter (identité déclarative), comme c'était le cas dans le web 1.0 (Georges, 2009, 2010; et al., 2009). Du web 1.0 au web dynamique, les interfaces se sont développées en valorisant une présentation éphémère du sujet qui n'existe que parce qu'il se manifeste aux autres en continu.

Ces résultats confirment la force du processus d'alterdocumentation: les données déclaratives sont émancipées du champ de maîtrise de l'utilisateur par une computation explicite du système. Les problèmes posés par ce phénomène sont non seulement la confidentialité des données personnelles, mais aussi l'intégrité de leur lecture par le système : le processus de recomposition et de computation des traces implique une grille 
interprétative implicite portée par l'interface ; elle valorise par exemple, dans Facebook, les pratiques de consommation du numérique (par exemple : avoir beaucoup d'amis dans sa boîte d'amis, de nombreux commentaires valorisants sur son mur, publiés par des personnes ayant une photographie jugée attrayante cf. Zywica et Danowski, 2008). Or, transférée dans le cadre des bibliothèques, la consommation est celle de produits culturels : dès lors, accumuler les commentaires d'ouvrages et les captures à visée de participation à la constitution de collections spécifiques permet de valoriser la facette culturelle voire créative de la présentation de soi. Par exemple l'outil de réseaux sociaux Academia.edu propose aux utilisateurs de se présenter par les articles qu'ils ont écrits mais aussi par leurs notes de lecture, de sorte que la relation sociale s'opère en partie par la mise à disposition de documents informatifs. Dans cet esprit de mise à disposition sur le modèle de la diffusion, les demandes d'amis ne sont pas le produit d'une procédure d'engagement-validation réciproque, comme c'est le cas dans la plupart des outils de réseaux sociaux (Linkedin, Facebook, Myspace), mais sur le principe du suivi comme dans l'outil Twitter ("Follow me ») de diffusion d'informations : la présentation de soi par le partage et la diffusion d'informations conduit à une communication sur le modèle du suivi/suiveur. Ce dispositif permet de modérer la part de consommation sociale implicite de nombreux outils du web 2.0 pour privilégier une modalité de relation fondée sur la curiosité et le désir d'être informé, plutôt que par la tentation du paraître.

Le modèle et les méthodes de mesure employées dans notre recherche ont permis de distinguer de grandes tendances comme celles du web social à dessaisir l'utilisateur de la maitrise de ses traces. Toutefois, cette méthodologie présente des biais relatifs à l'échantillonnage des informations. D'une part, les informations déclaratives sont bien des re-présentations de soi situées dans un environnement communicationnel, elles ne sont pas fiables : $30 \%$ des adolescents mentiraient sur leur âge, leur ville et leur statut social (Lenhart, 2007) : ce biais est propre à la plupart des présentations de soi informatisées. D’autre part, les fonctionnalités de gestion de la visibilité des informations émises (« être ami avec X » signifie « être visible pour X» dans Facebook) rend l'échantillonnage par recueil manuel des informations très subjectif, puisque seuls les amis d'une personnecible peuvent être observés : il est alors nécessaire d'intégrer à l'analyse le caractère participant de ce type d'échantillonnage, représentatif des personnes fréquentées par la personne-cible (Georges, 2009). Le recueil automatisé par création d'un programme spécifique de moissonnage d'informations ${ }^{2}$ (Georges et al., 2009) permet de contrecarrer ce biais, mais se heurte à un nouveau biais : les personnes donnant leur accord présentent une faible activité actuelle. Ce biais est propre à des systèmes conçus sur une procédure d'engagement-validation réciproque des amis (Linkedin, Facebook, Myspace), tandis que les systèmes fondés une procédure de suivi et de diffusion (Twitter, Adademia.edu) laissent les profils visibles par tous.

\section{Visualisation des données : SIC et Sciences Informatiques}

Pour visualiser les données recueillies, nous avons eu recours dans la partie de la recherche consacrée au changement de paradigme de représentation identitaire, à des graphes-radar. Dans un second temps, dans le cadre d'une collaboration avec le LIRMM, nous avons utilisé des graphes nœud-lien pour questionner la corrélation entre le 
comportement déclaratif et les activités de partage (partage social: groupes, amis communs ; partage de médias : photos cf. fig. 5).

Les graphes-radar (fig.4) ont pour qualité d'être d'une lecture intuitive et sont particulièrement adaptés à la représentation de l'identité numérique car ils permettent de visualiser une catégorie d'informations sur chaque axe, tout en signifiant bien par le croisement des axes en un point que chacun de ces axes participe de la caractérisation d'un même référent (un utilisateur ou un ensemble d'utilisateurs).

Figure 4 : Graphes « radar » de l'identité numérique. "Population totale » (ligne 1) : fréquence moyenne des utilisateurs hyper-visibles (trait continu épais) et des utilisateurs cachés (trait pointillé épais) ; graphes d'un utilisateur caché (ligne 2) et d'un utilisateur hyper-visible (ligne 3) (Georges, 2009 : 21).

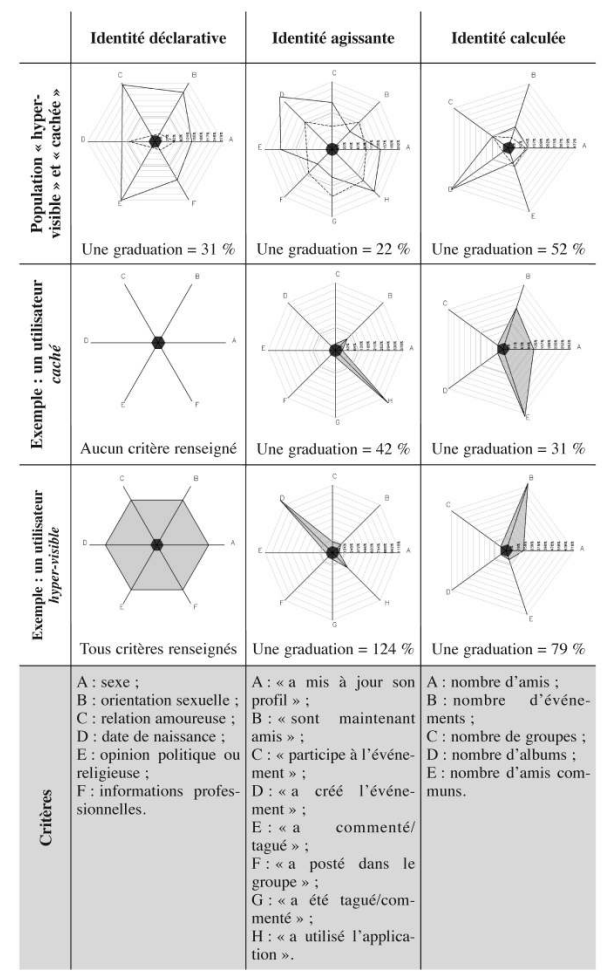

Les graphes nœud-lien sont utilisés dans le domaine de la visualisation de connaissances (Herman et al., 2000; Artignan et al., 2009) et pour visualiser les relations interindividuelles dans les réseaux sociaux en ligne (Mutton, 2004 ; White, $2008: 8,204$ ). En collaboration avec une équipe du LIRMM spécialisée en visualisation, nous avons choisi d'utiliser ces graphes pour visualiser la structuration du lien social par des éléments de l'identité calculée visibles sur les profils utilisateurs (nombre de groupes communs, d'amis communs et de tags photo). Les utilisateurs déclarant un grand nombre d'informations (hypervisibles) ont-ils tendance à partager des activités ou des documents avec d'autres utilisateurs hypervisibles? Le jeu de données et le nombre d'informations sont encore insuffisants pour produire une analyse qualitative solide (cf.: Georges et al., 2009), toutefois cet outil se distingue des outils de visualisation des relations d'amitié déclarées en proposant une analyse croisée, à la fois qualitative et quantifiée, des traces de partage : ainsi, le croisement de deux types de données (exemple fig. 5 : sociatifs et possessifs cf. Georges, 2009). 
Figure 5 : Vues de profils d'utilisateurs individuels (de a. à d.) et vue collective de 8 utilisateurs dans Facebook (e.) (Georges et al., 2009)
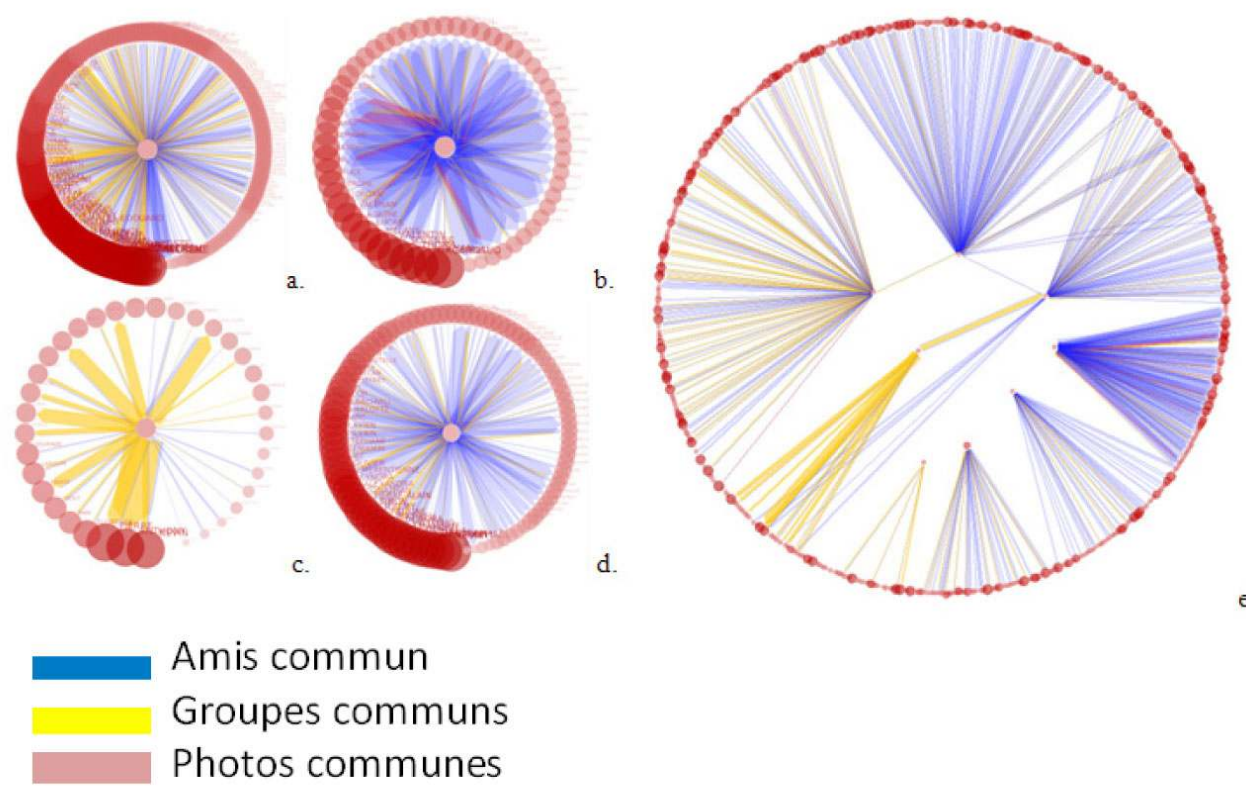

Epaisseur $=$ nombre

Notre projet, né de la construction d'un modèle et d'une approche quantifiée en SIC, s'est enrichi de la collaboration avec l'informatique par le développement d'outils pour l'analyse de l'identité numérique et de l'impact des protocoles sur les usages (fig. 6). La posture propre à chaque discipline s'est peu à peu précisée (Georges et al., 2010). L'informatique souhaite concevoir et mettre en place de nouveaux dispositifs technologiques: augmentation du nombre de profils visualisés, intégration d'un algorithme de fouille de données regroupant en clusters des profils similaires, utilisation d'autres indices visuels et de visualisations en 3D. Les SIC interviennent dans chacune de ces dimensions, en nourrissant le projet par l'orientation des questionnements sur les usages : définition du protocole d'expérimentation, choix des informations à visualiser, choix et recadrage des graphes, analyse. 


\section{Identification des besoins et mise en place de nouveaux dispositifs technologiques (langages, formats, applications)}

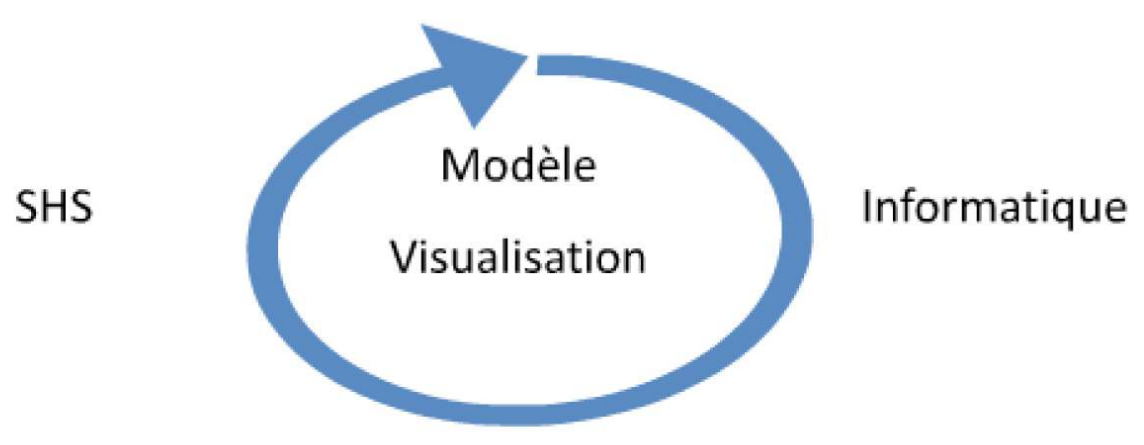

Analyse des productions et des usages

\section{Conclusion}

21 Cet article envisage l'intégration possible des réseaux sociaux en ligne dans les bibliothèques dans la perspective de la valorisation des collections, de l'ouverture à de nouveaux publics, de la participation des lecteurs à la documentarisation. Quels bénéfices présentent les outils de réseaux sociaux pour lecteur? Les relations entre les utilisateurs des réseaux sociaux en ligne se déploient via les informations présentées sur leur profil: en interagissant avec le dispositif et en commentant des ouvrages ou des médias, le lecteur-utilisateur construit son identité informatisée. La conception d'un projet d'intégration des réseaux sociaux dans les bibliothèques repose sur l'adéquation du dispositif aux usages et à la représentation identitaire du lecteur. La lecture synthétique d'un modèle de l'identité numérique et de son application quantifiée en utilisant les outils de visualisation, à la lumière de la notion d'alterdocumentation, dans le contexte des pratiques informationnelles des lecteurs, a mis en lumière des pistes en vue de la conception de dispositifs du web social adaptés aux bibliothèques et de l'analyse de leurs usages. D'une part, le partage de commentaires sur des ouvrages et la valorisation par les lecteurs de médias sur leur profils, considérés comme participant de la présentation sociale de soi, favoriseraient l'investissement personnel dans ces activités par leur intégration dans une dynamique de construction de l'identité sociale informatisée. D'autre part, le typage des liens interpersonnels sur le modèle du suivi-suiveur (Twitter, Academia.edu) plutôt que sur le modèle de la validation réciproque (Linkedin, Facebook) impliquerait quant à lui un processus de communication interpersonnel propice au partage des connaissances. 


\section{BIBLIOGRAPHIE}

Artignan, G., Hascoët, M. et Lafourcade, M., (2009), Multiscale Visual Analysis of Lexical Networks, IV'09: $13^{\text {th }}$ International Conference Information Visualisation, July 15-17, 685-690.

Chaudiron, S. et Ihadjadene, M., (2002), Quelle place pour l'usager dans l'évaluation des SRI ?, in Recherches récentes en Sciences de l'Information, convergences et dynamiques, Actes du colloque MICS-LERASS, Toulouse, ADBS Éditions, 21-22 mars 2002, 211-233.

Choo, C. W., (1996), The Knowing Organization, How organizations use information to construct meaning, create knowledge and make decision, International journal of information management, Vol. 16, n 5, 329-340.

Ertzscheid, O., (2009), L'homme, un document comme les autres, Hermès, $\mathrm{n}^{\circ}$ 53, CNRS Éditions, Paris, 33-40, $1^{\text {ére }}$ version consultable dans Archives SIC : L'homme est un document comme les autres : du World Wide Web au World Life Web : sic_0037745.

Georges, F., (2009), Identité numérique et Représentation de soi : analyse sémiotique et quantitative de l'emprise culturelle du web 2.0, Réseaux, Vol. 2, n 154, 165-193.

Georges, F., Seilles, A., Artignan, G., Arnaud, B., Rodriguez, N., Hascoët, M., Sallatin, J. et Dresp-Langley, B., (2009), Sémiotique et visualisation de l'identité numérique : une étude comparée de Facebook et Myspace, Actes de la conférence H2PTM'09, Paris, Hermès, 257-268.

Georges, F., Seilles, A. et Sallantin, J., (2010), Sciences de l'information et de la Communication et Sciences Informatiques : vers une approche quantifiée de la Représentation de soi dans la Communication Médiée par Ordinateur, $17^{\mathrm{e}}$ Congrès de la SFSIC, 23-26 juin, Dijon.

Henneron, G., Metzger, J. et Polity, Y., (1997), Information et activité professionnelle, Rapport Intermédiaire en sciences sociales et humaines, Ersico, Univ. Lyon 3.

Herman, I., Melançon, G. et Marshall, M. S., (2000), Graph visualization and navigation in information visualization : A survey, IEEE Transactions on Visualization and Computer Graphics, Vol. 6, $n^{\circ} 1,24-43$.

Lenhart, A. et Madden, M., (2007), Teens, Privacy \& Online Social Networks, Pew Internet \& American Life Project, Washington.

Mutton, P., (2004), Inferring and Visualizing Social Networks on Internet Relay Chat, Proceedings of Information Visualisation IV'04, 35-43.

Sarméjeanne, V., (2001), La recherche d'information pour l'enseignement, Thèse de doctorat, Univ. Lyon 3.

Silipigni Connaway, L. et Dickey, T., (2010), The Digital Information Seeker : Report of the Findings from Selected OCLC, RIN, and JISC User Behaviour Projects, OCLC Research.

Suraweera, N., Razali, N., Bahadur Chouhan, L., Tamang, N., Hubilla, A., Ratnayake, M., Rubosa, N., Akbar Malik, F. et Mahesar, S., (2010), Value of Social Networking in Libraries and Information Organizations in Asia and Oceania, World library and information congress : $76^{\text {th }}$ IFLA general conference and assembly, 10-15 August 2010, Gothenburg, Sweden.

Thivant, E. et Bouzidi, L., (2006), Les pratiques informationnelles pour les financiers, Information Seeking In Context ISIC 2006, 18-21 juillet, Sydney, Australie. 
University College London (UCL) CIBER group, (2008), Information behaviour of the researcher of the future, London, University College London, CIBER Briefing paper.

Walther, J., Van der Heide, B., Kim, S., Westerman, D. et Tom Tong, S., (2008), The role of friends'appearance and behavior on evaluations of individuals on Facebook: Are we known by the company we keep ?, Human Communication research, $\mathrm{n}^{\circ} 34,28-49$.

White, C., (2008), Identity and Control : How Social Formations Emerge, Second edition, Princeton university press, 2008.

Zywica, J. et Danowski, J., (2008), The Faces of Facebookers : Investigating Social Enhancement and Social Compensation Hypotheses ; Predicting Facebook and Offline Popularity from Sociability and Self-Esteem, and Mapping the Meanings of Popularity with Semantic Networks, Journal of Computer-Mediated Communication, Vol. 14, Issue 1, 1-34.

\section{NOTES}

1. Par exemple, le département Audiovisuel de la BNF comporte des collections "Art numérique » et " Jeu vidéo ».

2. Le recueil automatisé d'informations est rendu possible par la mise à disposition dans Facebook d'interfaces de programmation (API Application Programming Interface) destinés au relevé de certaines informations sur les profils. Il ouvre des perspectives de recueil massif de données, par opposition au recueil manuel.

\section{RÉSUMÉS}

Cet article envisage l'intégration possible des réseaux sociaux en ligne en bibliothèque et questionne ses enjeux dans les pratiques informationnelles et les identités documentaires des lecteurs. La lecture d'un modèle de l'identité numérique à la lumière de la notion d'alterdocumentation fait apparaître des pistes pour la conception de tels dispositifs et l'analyse des usages. Le partage de documents favoriserait l'engagement des lecteurs par sa participation au processus de présentation de soi informatisée ; le modèle du lien interpersonnel suivi/suiveur serait plus favorable au développement des activités de partage de connaissances que le modèle de la validation réciproque.

This paper examines the possible integration of online social networks into libraries and their consequences on information practices and digital identities. Focusing on digital identities and resorting to the notion of "alter-documentation" offers new avenues for the design and use analysis of such mechanisms. Document-sharing favors reader commitment as it participates in the process of digital self-presentation. The model of followed/follower interpersonal relationships is more conducive to knowledge-sharing activities than the model of mutual validation. 
INDEX

Mots-clés : communication médiée par ordinateur, identité numérique, document, représentation de soi, pratique informationnelle, données personnelles, réseau social, partage de médias

Keywords : computer mediated communication, digital identity, document, information practice, self-representation, personal data, media sharing, social network

\section{AUTEUR}

\section{FANNY GEORGES}

LTCI (CNRS UMR 5141) - Telecom ParisTech

Fanny Georges est chercheure en sciences de la communication (Telecom Paritech). Ses recherches portent sur les représentations identitaires informatisées. Elle est l'auteur de l'ouvrage Identités virtuelles, les profils utilisateur du Web 2.0, paru aux éditions Questions théoriques en 2010. Adresse électronique : fannygeorges@free.fr. 